\title{
Cold winds and economic recession
}

At the time of writing the snow is falling and the weather is bitterly cold; but the cold North Easterly is nothing to the biting wind of economic recession that grips Ireland at the minute! Unprecedented cuts in healthcare and higher education spending have been announced in the 2010 budget. $^{.}$Cuts of $€ 4 \mathrm{bn}$ from public spending will have a major impact on physiotherapy provision, jobs, education and patient care.

In this harsh economic climate how should physiotherapists and the physiotherapy profession respond? Should we hunker down, hold what we have and wait for better times to come. No, now more than ever, physiotherapists and the physiotherapy profession need to proclaim, and more essentially, to demonstrate, the positive impact we have on patient care and patient outcomes. The argument is often made that evaluating practice and measuring outcomes requires quality research which is extremely costly and time intensive. Research is indeed costly and time consuming; however, I would contest the premise that this represents an insurmountable barrier to practice evaluation.

In the current issue of the Journal, Kennedy and colleagues present the findings of an audit of physiotherapy service for patients following neck dissection for head and neck cancer. These authors have demonstrated, using validated outcome measures, significant improvements in patient outcomes and quality of life. This audit is valuable and provides initial evidence of the impact physiotherapy service has for this important and vulnerable patient group. Hopefully Kennedy and her colleagues will use these preliminary finding as the basis for robust research in this area.

Crowley has also explored the impact of physiotherapy intervention for patients attending a cardiac rehabilitation programme. Using a mixed methodological approach, Crowley has demonstrated improvements in patient outcomes particularly from the patients' perspective. Again an important demonstration of the impact physiotherapy can make to patient care. Examples such as these (and I am sure there are may more examples going on in every area of physiotherapy) can, and should, be used to support the essential role physiotherapy plays in health care.

Walsh et al and O'Sullivan et al in the current issue also address practice evaluation, but perhaps at a more fundamental level. In order to claim that our physiotherapy interventions are effective, we must be able to clearly demonstrate that the measures we use are valid, reliable and without bias. Both these groups of researchers have evaluated the inter-rater and intra-rater reliability of different outcome measures. The specific study design and outcome measures used are subject specific and are less important than the approach taken. O'Sullivan et al and Walsh et al have both used the most rigorous statistical analysis to determine the inter-rater and intra-rater reliability, both groups using interclass correlation coefficients and Bland and Altman limits of agreement. The Bland and Altman paper ${ }^{2}$ reporting this method of comparing two methods of measurement is one of the most highly cited statistical papers ever published (currently approximately 17914 citations!) and the most highly cited paper in the history of The Lancet. ${ }^{3}$ Without access to extensive grant funding these authors have been able to systematically evaluate their selected outcome measures and evaluate their use in clinical practice. Having a rigorous approach to the evaluation of the measurement of outcomes is no more expensive than a less rigorous approach and is certainly 'doable' within routine clinical practice.

This issue also includes two interesting papers on low back pain. Those of us in physiotherapy education will read with interest Horrell et al's paper on the reported prevalence of low back pain in undergraduate students (including physiotherapy students) attending the University of Plymouth. While it could be argued that questions around the management of low back pain have been fairly comprehensively addressed in the literature, ${ }^{4}$ implementing the recommendation in the various guidelines is perhaps more problematic. Cunningham et al's paper explores some of barriers and facilitators to implementing low back pain guidelines in the working environment, an important next step in the management of this perennial problem.

JG McVeigh PhD DipOrthMed BSc(Hons)MCSP

School of Health Sciences

University of Ulster

Jordanstown

Northern Ireland

Email: j.mcveigh@ulster.ac.uk

\section{REFERENCES}

1. Financial statement of the minister for finance Mr. Brian Lenihan, T.D. 9 December 2009. [cited 10 January 2010] Available from: http://www.budget.gov.ie/Budgets/2010/Documents/FINAL\%20Speech. $\mathrm{pdf}$

2. Bland J, Altman D. Statistical Methods for Assessing agreement between two methods of clinical measurement. Lancet. 1986;1:307-10.

3. Sharp D. As we said. Lancet 2004; 364:744

4. Bouwmeester W, van Enst A, van Tulder M. Quality of low back pain guidelines improved. Spine. 2009;34:2562-7. 